\title{
Is anatomical distribution helpful for differentiating TB spondylitis from neoplastic causes of extradural spinal cord compression in children? A pilot study
}

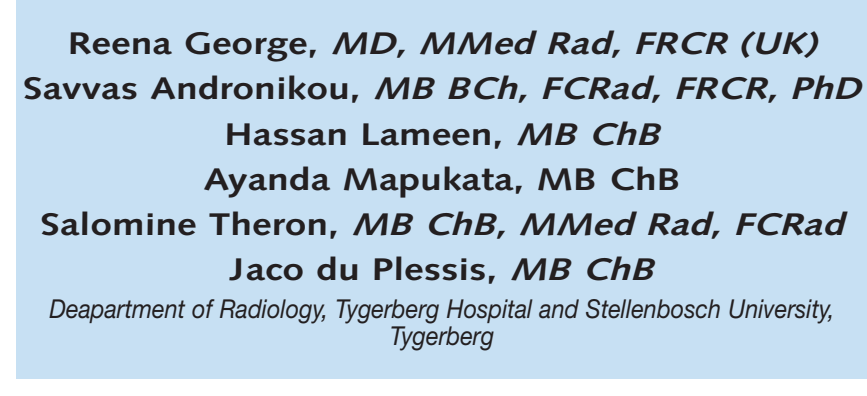

\section{Abstract}

Background. Extradural spinal cord compression in children may be the result of infection/inflammation or neoplasia. It is vital to differentiate between the two as there is considerable difference in the management of these entities.

Objective. The aim of this paper is to determine whether there are significant differences between TB spondylitis and neoplasia causing extradural compression of the cord with regard to the anatomical distribution and compartmentalisation.

Materials and methods. A group of 16 children between the ages of 4 months and 14.2 years who had magnetic resonance (MR) evaluation of the spine consecutively were analysed retrospectively.

Results. A wide variety of neoplasms demonstrated an anatomical site and compartment on MRI that is 'characteristic' of TB spondylitis.

Conclusion. Anatomical distribution alone does not assist in narrowing the differential diagnosis. There is still need for biopsy in children with extradural compression by a mass.

\section{Introduction}

Extradural spinal cord compression may be the result of infection, inflammation or neoplasia. Masses may arise from the thoracic and abdominal cavities, from the vertebral column (and discs) as well as both sympathetic and exiting nerves. Categorisation of lesions according to the level involved (cervical, thoracic, lumbar, sacral) and according to the compartments involved, in combination or singly (extraspinal, vertebral, intraspinal extradural, intradural extramedullary and intradural intramedullary), is routinely performed with the aim of narrowing the differential diagnosis. ${ }^{1}$ In particular, in the developing world (and with the advent of HIV in the developed world), it is necessary to distinguish TB spondylitis from neoplastic causes, to avoid biopsy. ${ }^{2} \mathrm{~TB}$ spondylitis is treated medically in most cases ${ }^{2}$ while neoplastic lesions require any combination of surgery, chemotherapy and radiotherapy to alleviate the cord compression. Using signal characteristics on magnetic resonance imaging (MRI) to distinguish infective lesions from neoplasia does not usually resolve the differential diagnosis, with the majority of lesions (infective and neoplastic) demonstrating low or intermediate signal on $\mathrm{T} 1$ and high signal on T2 with abscess formation in TB spondylitis ${ }^{2,3}$ and cyst formation in some neoplasms (Ewing's sarcoma, neuroblastoma) ${ }^{4,5}$ Rim enhancement of the soft-tissue mass is a feature that most strongly supports a diagnosis of TB spondylitis ${ }^{2,3}$ but this is also seen in pyogenic spondylitis ${ }^{5}$ and may occur when enhancing neoplasms show cystic degeneration. ${ }^{5}$ Involvement of a disc in conjunction with a vertebral body is considered a strong pointer to an infective spondylitis $^{2}$ but has been shown with metastatic disease ${ }^{3}$ and other neoplasms. Subligamentous spread is seen in TB spondylitis demonstrating extension above and below the level of involvement but this extension is also seen with neuroblastoma ${ }^{5}$ and lymphoma involving the spinal canal. The aim of this paper is to determine whether there are significant differences between TB spondylitis and neoplasia causing extradural compression of the cord with regard to the anatomical distribution and compartmentalisation without considering the signal characteristics or contrast enhancement.

\section{Aim}

The aim was to determine if the demonstration of anatomical distribution/compartmentalisation on magnetic resonance imaging (MRI) can differentiate TB spondylitis from neoplasia in extradural cord compression in children.

\section{Materials and methods}

A subgroup of patients who had a diagnosis of extradural spinal cord compression was extracted from a database of MRI scans performed of the spine in children at one institution over one year. Incomplete or inadequate studies, inadequate clinical notes and patients imaged for trauma or congenital anomalies (such as tethered cord/dysraphism) were excluded. A paediatric radiologist and a registrar, both blinded to the final diagnoses, reviewed images. Features recorded included vertebral level involved (cervical, thoracic, lumbosacral), uni- or multifocal involvement, involvement over numerous vertebral levels and the compartments involved in combination (extraspinal, vertebral, intraspinal extradural). The final diagnoses reached through biopsy, trial of therapy and associated clinical findings were used to categorise the patients after the blinded CT review into a 'TB spondylitis group' and a 'neoplastic group'. Only the anatomical distribution and compartment information was then used to determine differences between the two groups.

\section{Results}

From a total of 67 patients who had undergone MRI of the spine, 16 had a diagnosis of extradural spinal pathology (24\%) and were included in the study. There were 9 males and 7 females and ages ranged from 
4 months to 14 years and 2 months (average 97 months/8 years and 1 month). Final diagnosis comprised 8 cases of neoplastic disease, 6 cases of infection and 2 cases of inflammation. The two cases of non-specific inflammation were excluded for the purposes of this study. Neoplastic lesions included neurofibromas (2), ganglioneuroma (1), lymphoma (1), pulmonary blastoma (1) (Fig. 1), Ewing's sarcoma (1) (Fig. 2a \& b), infantile fibromatosis (1) (Fig. 3a \& b) and haemangioma (1) (Fig. 4). Table I compares the 2 groups. The site was primarily thoracic for both groups and cervical location was uncommon for TB. Both groups involved multiple vertebral levels in contiguity (TB 6/6, neoplasm 7/8) but TB was often multifocal (6/6) whereas no neoplastic process was seen multifocally. Both TB and neoplasia involved the extraspinal compartment regularly (TB 4/6, neoplasia 6/8) and TB always involved the vertebral body (6/6) while neoplasia only involved the vertebral body in half the cases $(4 / 8)$.

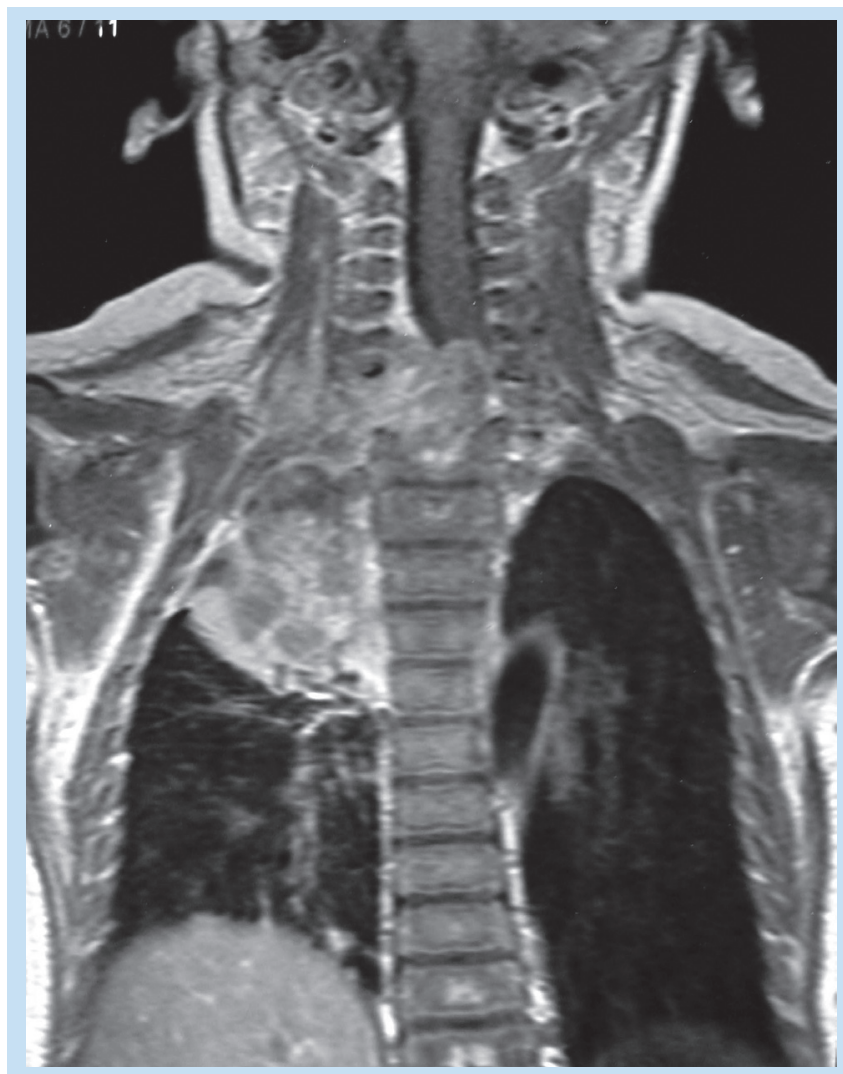

Fig 1. A 4-month-old boy with pulmonary blastoma - coronal T1-weighted post-gadolinium-enhanced image shows the mass extending into the spinal canal with a large extradural component.

\section{Discussion}

It is reported and taught that an effective and commonly used approach to interpretation of disease involving the spine is to categorise the disease as extradural, intradural extramedullary or intradural intramedullary. ${ }^{1}$ This is best achieved using axial and sagittal MRI sequences. ${ }^{1}$ The intent is to narrow the differential diagnosis in the hope of reaching an imaging diagnosis, thereby avoiding biopsy in addition to planning surgery/management in the case of neoplasia. The pattern of bone and disc involvement is insufficient grounds for differentiating TB spondylitis
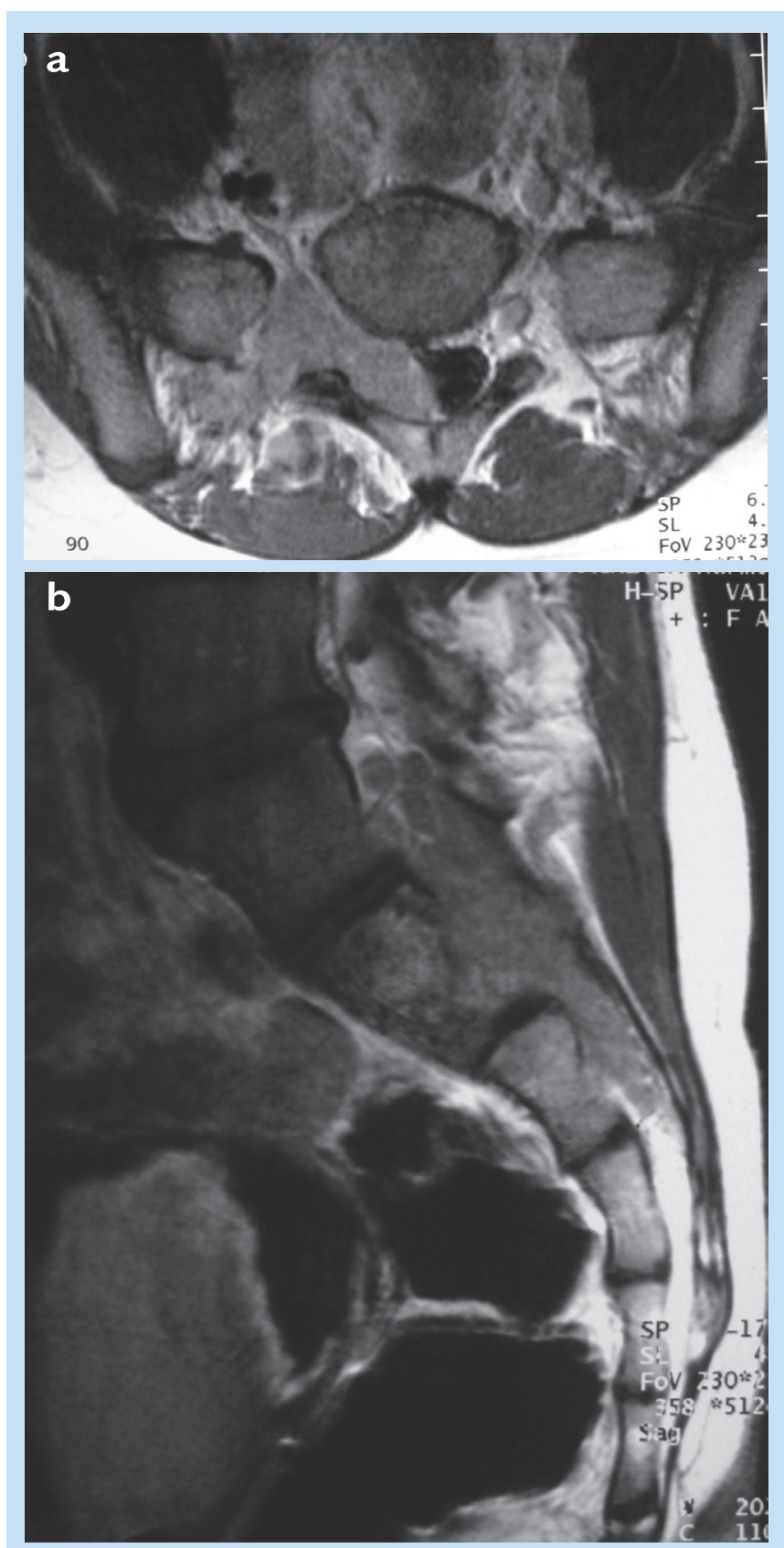

Fig. 2. An 11-year-old girl with Ewing's sarcoma of the sacrum Axial (a), and sagittal (b) T1-weighted gadolinium-enhanced images. Extradural extension of the iso-intense mass through the neural foramen.

from metastatic disease in adults ${ }^{3}$ and this is also sometimes the case with paediatric imaging. Multiple destructive vertebral bone lesions in children other than TB spondylitis include: (i) malignancies, particularly neuroblastoma metastases, lymphoma and Ewing's sarcoma; (ii) benign bone tumours such as aneurysmal bone cysts; and (iii) infections including pyogenic, Brucella, fungal and echinococcal lesions. ${ }^{2}$ By far the most common diagnosis in the developing world is infective spondylitis. ${ }^{2}$ Although computed tomography (CT) and MRI features of TB spondylitis have been reported in adults and children there is significant overlap with neoplastic disease. ${ }^{2,3}$ 


\section{ORIGINAL ARTICLE}

\begin{tabular}{|lll|}
\hline \multicolumn{2}{|c|}{ Table I. Comparison of TB spondylitis } & with neoplasia for anatomical distribution \\
\hline Age & TB spondylitis (6) & Neoplasia (8) \\
Male & 109.8 months & 87.5 months \\
Female & 2 & 6 \\
Cervical & 4 & 2 \\
Thoracic & 1 & 3 \\
Lumbar & 5 & 4 \\
Sacral & 1 & 3 \\
Multiple vertebral segments (contiguous) & 1 & 2 \\
Multifocal involvement (distant) & 6 & 7 \\
Extraspinal with intraspinal extradural & 3 & 0 \\
Vertebral with intraspinal extradural & 4 & 6 \\
& 6 & 4 \\
\hline
\end{tabular}
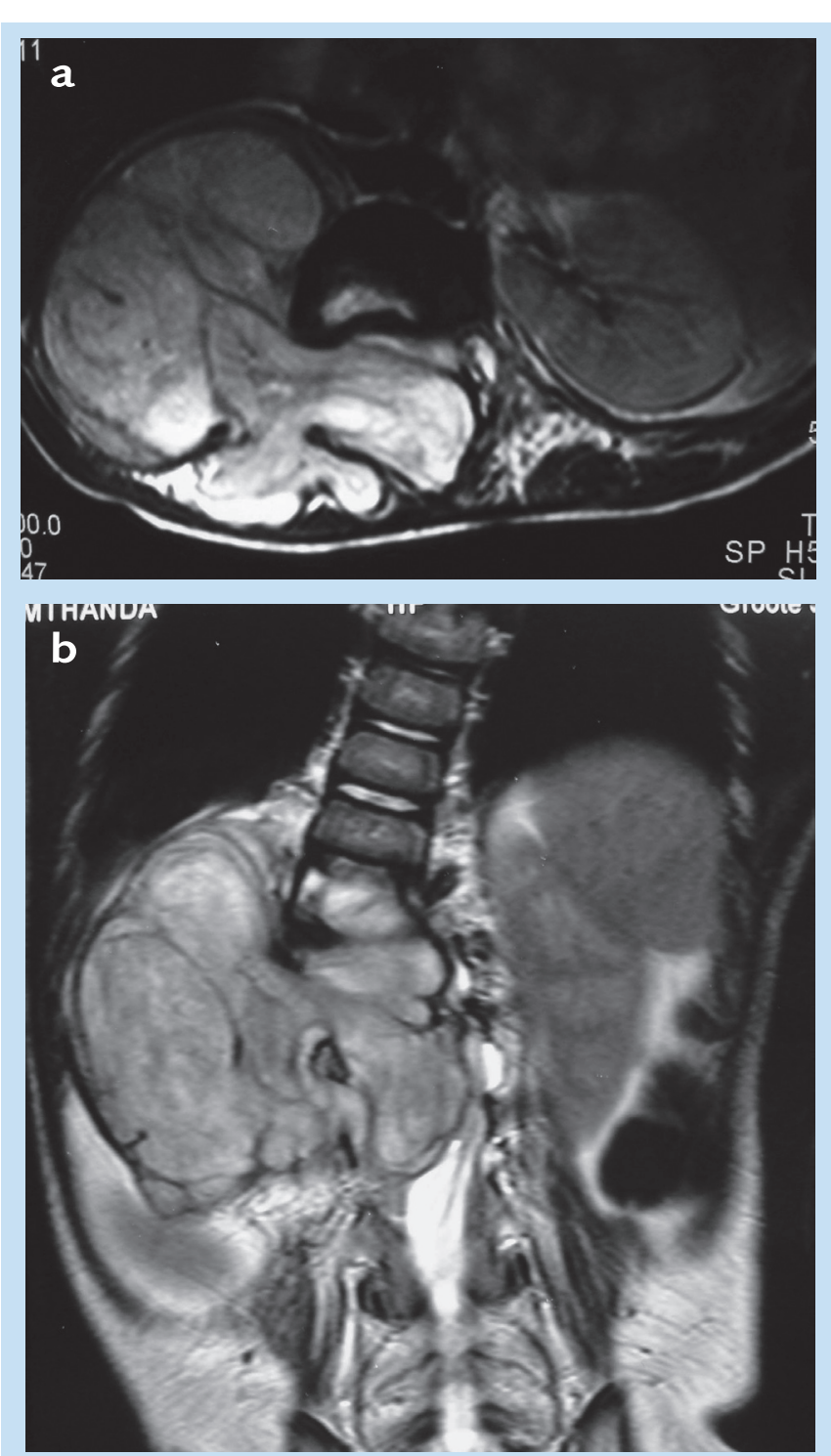

Fig. 3. A one-and-a-half-year-old boy with infantile fibromatosis. Axial (a) and coronal (b) T2-weighted studies show a hyperintense mass with extradural extension via the neural foramina.

\section{Neoplasms}

Extradural neoplasms in children are usually an extension of neoplasms in the paravertebral soft tissues or anterior vertebral column, most commonly neuroblastoma. ${ }^{6}$

Neuroblastoma, ganglioneuroblastoma and ganglioneuroma arise wherever there is sympathetic tissue. ${ }^{5} \mathrm{MRI}$ is the preferred modality for demonstrating intraspinal extension of the primary tumour through the neural foramina - dumbbell neuroblastoma. ${ }^{5,6}$ This occurs in $10 \%$ of abdominal and $28 \%$ of thoracic neuroblastomas. ${ }^{5}$ Neuroblastoma may also result in vertebral body metastases, which can also result in cord compression.

Lymphoma may involve the paravertebral lymph nodes and cause adjacent vertebral invasion, may show malignant vertebral infiltration with or without intra- and para-spinal involvement, may involve the epidural space in isolation and may present as an intramedullary mass. Spinal cord compression as the presenting feature is rare however (0.1$3.3 \%$ ). It is less frequent in childhood than in adulthood and usually affects the cervical region. When spinal localisation is the sole manifestation of lymphoma, the imaging characteristics are not sufficient to make a diagnosis and it is reported that this can only be made by means of biopsy.

Eosinophilic granuloma lesions affect the vertebral body primarily but may extend into the adjacent epidural space to compress the thecal sac. $^{6}$

Ewing sarcoma (primitive neuroectodermal tumour) often originates in the paravertebral soft tissue and extends through the neural foramina, but may also infiltrate other surrounding structures. ${ }^{4}$

Spinal tumours form part of the spectrum of both neurofibromatosis I and II and occur in a large number of patients in all segments of the spine. These are often intraforaminal (57\%) and are surrounded by soft-tissue tumour. ${ }^{8,9}$

\section{Infection}

A common location for infection in the paediatric spine is the anterior vertebral column; this may involve the vertebral body in isolation, the vertebral body and intervening disc, or the disc alone. ${ }^{6}$ Infection may extend into the epidural space causing compression of the thecal sac. ${ }^{6}$

The imaging features characteristic of TB spondylitis in addition to 


\section{ORIGINAL ARTICLE}

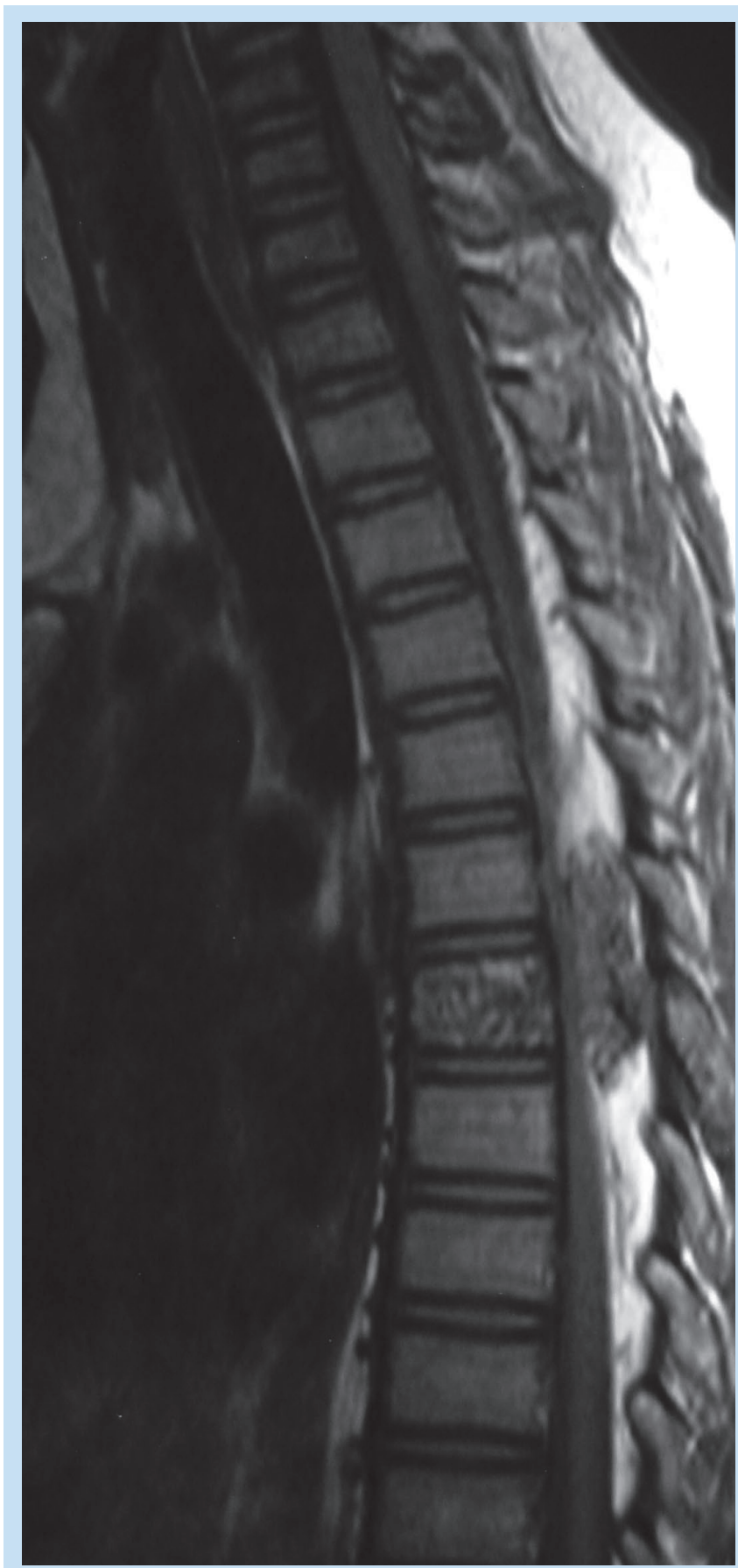

Fig. 4. A 10-year-old boy with capillary haemangioma of the vertebral body. Sagittal T1-weighted image shows a mottled pattern of increased intensity with a large extradural component.

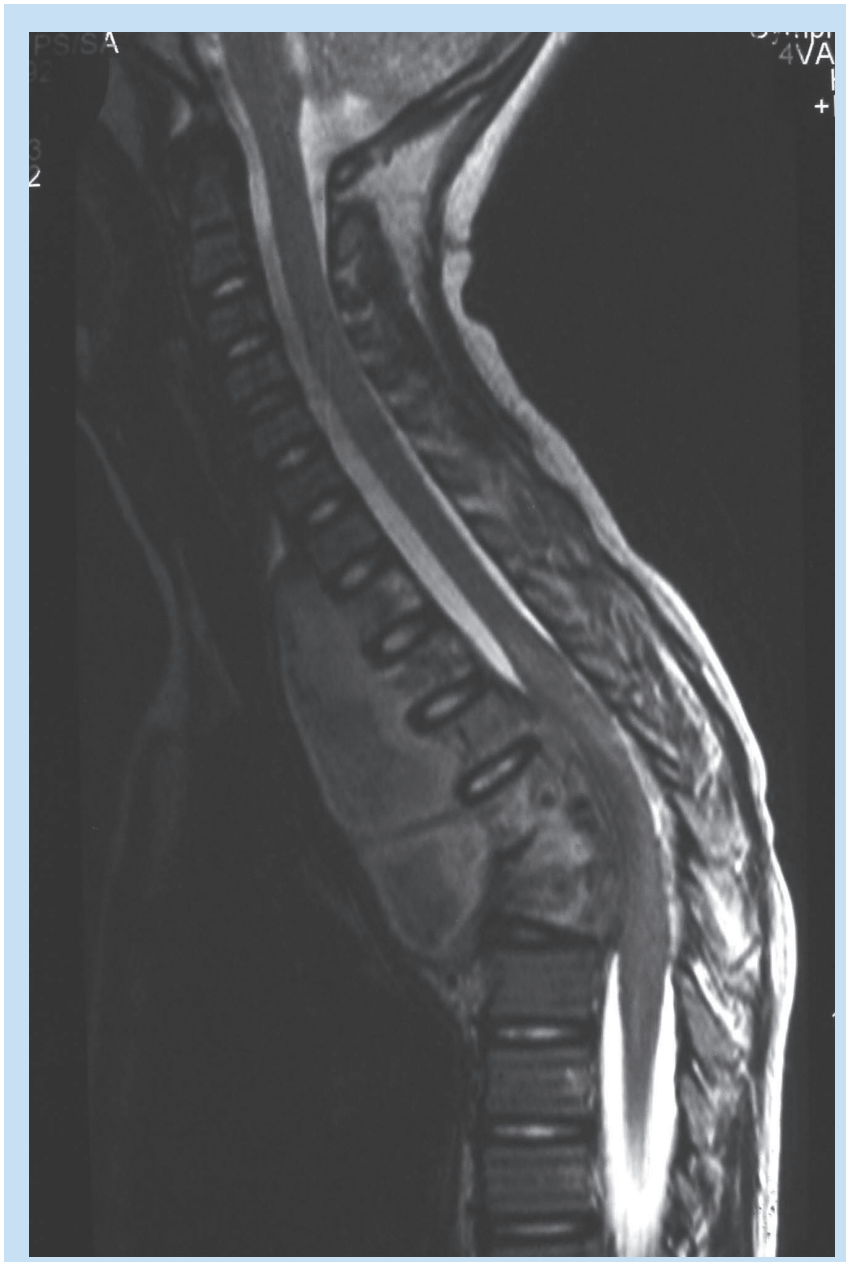

Fig. 5. A 12-year-old boy with TB spondylitis. Sagittal T2-weighted image shows contiguous vertebral body and disc destruction with a large anterior mass and a posterior extradural component compressing the spinal cord.

vertebral body involvement include involvement of multiple vertebral segments (contiguously or far apart), presence of a pre/paravertebral mass/abscess; rim enhancement of the abscess; involvement of the disc and subligamentous spread above and below the level of bone destruction (Figs 5, 6a \& b). ${ }^{2,3}$ The thoracolumbar region is reported to be the commonest involved. ${ }^{2,3}$ One author reports that bony fragments (low signal on all sequences) only occur with $\mathrm{TB},{ }^{3}$ but the other characteristics listed above may be mimicked by neoplastic disease (Table II).

\section{Conclusion}

A small number of patients were evaluated in this paper, but even with this small sample a wide variety of neoplasms demonstrated features

\begin{tabular}{|ll|}
\hline \multicolumn{1}{|c|}{ Table II. Neoplastic mimickers of characteristic features of TB spondylitis } \\
\hline TB spondylitis characteristic & \multicolumn{1}{c|}{ Neoplastic mimicker } \\
\hline Pre/paravertebral abscess & Ewing's cystic degeneration \\
Rim enhancement of abscess & Cystic neuroblastoma/Ewing $\mathrm{s}^{5,4}$ \\
Extension beyond level of bone destruction & Neuroblastoma $^{5}$ \\
Multifocal & Neurofibromatosis $^{8}{ }^{8}$ neuroblastoma $^{5}$ \\
Disc involvement & Metastases $^{3}$ \\
& \\
\hline
\end{tabular}




\section{RIGINAL ARTICLE}
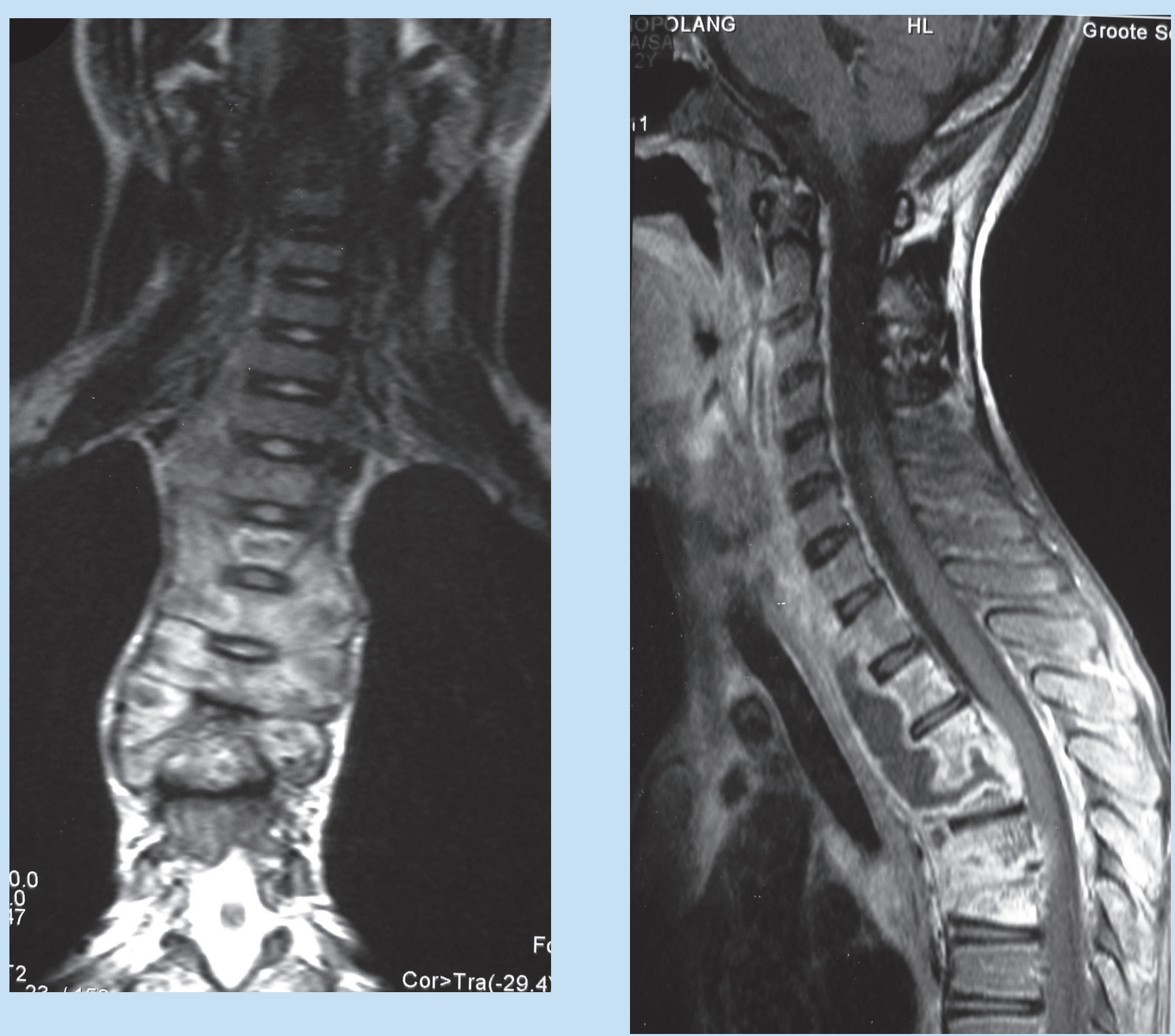

Fig. 6. A 10-year-old boy with TB spondylitis. (a) Sagittal T1 post-gadolinium and (b) coronal T2-weighted images show multiple contiguous vertebral bodies which have abnormal signal and enhancement as well as disc destruction. There is a predominantly anterior ring-enhancing soft-tissue mass but the smaller posterior extradural component causes spinal cord compression.

on MRI with regard to their anatomical site and compartment that are characteristic of TB spondylitis. It is not likely that anatomical distribution alone will assist in narrowing the differential diagnosis and obviate the need for biopsy in children with extradural compression by a mass. More research into new MRI sequences and use of tissue characterisation as well as gadolinium enhancement patterns are necessary to reach this goal, as current signal and enhancement characteristics for ТВ spondylitis and neoplasia also overlap.

1. Khanna AJ, Bruce A, Paul D. Magnetic resonance imaging of the pediatric spine. Journal of the American Academy of Orthopedic Surgeons 2003; 11: 248-259.

2. Andronikou S, Jadwat S, Douis $\mathrm{H}$. Patterns of disease on MRI in 53 children with tuberculous spondylitis and the role of gadolinium. Pediatr Radiol 2002; 32: 798-805.

3. Gupta RK, Agawal P, Rastogi H, Kumar S, Phadke RV, Krishnani N. Problems in distinguishing spinal tuberculosis from neoplasia on MRI. Neuroradiology 1996; 38: S97-S104

4. Tateishi U, Gladish G, Kusumoto M, et al. Chest wall tumours: radiologic findings and pathologic correlation. Radiographics 2003; 23: 1491-1508.

5. Lonergan GJ, Schwab CM, Suarez ES, Carlson CL. Neuroblastoma, ganglioneuroblastoma and ganglioneuroma: radiologic-pathologic correlation. Radiographics 2002; 22: 911-934.

6. Prenger E. Magnetic resonance imaging of the pediatric spine. Semin Ultrasound, CT MR 1991; 12(5): 410-428.

7 Acquaviva A, Marconcini S, Municchi G, Vallome I, Palma I. Non-Hodgkin lymphoma in a child presenting with acute paraplegia; a case report. Pediatr Hematol Oncol 2003; 20: 245-251.

8. Thakkar SD, Feigen U, Mautner VF. Spinal tumours in neurofibromatosis type I: an MRI study of frequency, multiplicity and variety. Neuroradiology 1999; 41: 635-629.

9. Mautner V-F, Tatagiba M, Lindenau M, et al. Spinal tumours in patients with neurofibromatosis type II: MR imaging study of frequency, multiplicity and variety. AJR 1995; 165: 951-955. 